\title{
The Analysis of a Piezoelectric Bimorph Beam with Two Input Base Motions for Power Harvesting
}

\author{
M F Lumentut, I M Howard \\ Theoretical and Applied Mechanics, Department of Mechanical Engineering, Curtin \\ University of Technology, Australia
}

E-mail: m.lumentut@postgrad.curtin.edu.au,i.howard@exchange.curtin.edu.au

\begin{abstract}
The exploitation of usable power from vibration environments shows potential benefit for recharging batteries and powering wireless transmission. In this paper, we present a novel technique for simulating the electromechanical cantilevered piezoelectric bimorph beam system with two input base transverse and longitudinal motions for predicting power harvesting. The piezoelectric bimorph beam with tip mass was modelled using the EulerBernoulli beam assumptions. The strain fields from transverse bending and longitudinal forms can affect the physical behaviour of the polarity and electric field in terms of the series and parallel connections of the piezoelectric bimorph, in such way that each connection has two vector configurations of X-poling and Y-poling due to input base motions. This situation must be correctly identified to form the piezoelectric couplings. The piezoelectric couplings can create the electrical force and moment of each piezoelectric layer in the mechanical domain. At this point, we introduce a new method of modelling the piezoelectric bimorph beam under two input base-motions using coupling superposition of the elastic-polarity field for predicting power harvesting. The constitutive dynamic equations were derived using the weak form from the Hamiltonian theorem, with Laplace transforms being used to obtain the multi-mode frequency response functions (FRFs) relating the input mechanical vibrations with the output dynamic displacement, velocity and power harvesting. The power harvesting predictions under parallel connection at frequencies close to the fundamental bending frequency demonstrate a possibility of being able to produce around $0.4 \mathrm{~mW}$ per unit input base transverse acceleration of $3 \mathrm{~m} / \mathrm{s}^{2}$. Furthermore, it is shown that varying the load resistance from $20 \mathrm{k} \Omega$ to $200 \mathrm{k} \Omega$ affects the amplitude of power harvesting as well as resulting in a shift of the first natural frequency from $76 \mathrm{~Hz}$ to $79 \mathrm{~Hz}$.
\end{abstract}

\section{Introduction}

In the last few years, the investigation of energy conversion techniques utilising the ambient vibration environment has been of great interests for many researchers. The energy extracted from the vibrating environments can be utilised for powering an electronic circuit as it can be capable of supplying direct current into a rechargeable battery or electrical power storage device. It can then be subsequently used for powering wireless sensor communication. The common energy harvester techniques can be achieved by using electromagnetic [1], electrostatic [2] and piezoelectric [3] transductions. Although, there have been numerous research publications to discuss the energy harvesting development, the study of piezoelectric harvester has been of great interest for many researchers because the piezoelectric component has potential benefit in the applications of micro-scale designs, compact 
configuration, high sensitivity with respect to low vibration and practical to use. In comparison, the electromagnetic devices have complex design configurations for the magnet and coil components and also need high vibration velocities to create high magnetic flux in order to obtain electric voltage as shown by Faraday's law. It also tends to be incompatible for micro-scale use. Moreover, the electrostatic concept can be compatible to micro-scale but has complex design configurations for the two plate capacitor components and requires the use of external voltages.

The concept of the electromechanical piezoelectric power harvester has wide ranging applications with current research emphasis investigating new theoretical and mathematical developments along with experimental studies. The extensive review of piezoelectric power harvester with different application models have been discussed by [3,4]. The experimental study of different types of piezoelectric components bonded onto cantilevered aluminium beam is given in [5]. Moreover, the mathematical and experimental developments of the piezoelectric bimorph beam based on input transverse excitation have been of major interest for investigating novel power harvester models. Extensive modelling of the piezoelectric bimorph beam using the Rayleigh-Ritz method with variance of load resistances under short and open circuits has been provided in [6], the optimal power harvester with single mode formulation in [7], and the optimisation design of different shapes of piezoelectric power harvesters in [8]. Recent theoretical and experimental models of the piezoelectric bimorph beam with input transverse excitation was also investigated using the frequency response function with varying load resistances [9].

In this paper, we propose the novel theoretical study of the electromechanical dynamic equation of the piezoelectric bimorph beam using the weak form of Hamiltonian's principle. In this model, the multi-mode frequency responses obtained using Laplace transformation were also developed to give the electromechanical dynamic relationships between two input base transverse and longitudinal excitations with multi output dynamic responses including power harvesting. In this case, we also present sample validation results between the theoretical and experimental studies of the frequency response analysis due to input base transverse excitation of the bimorph with parallel connections.

\section{Mathematical Formulations}

The electromechanical dynamic equations of the piezoelectric bimorph under two input base transverse and longitudinal excitations were formulated using the weak form of Hamiltonian's theorem using the strain energy of the central bimorph substructure (brass shim), the electrical enthalpy of the upper and lower piezoelectric layers, and the kinetic energy of the bimorph including the tip mass $[10,11]$. The constitutive equation can be stated after simplifying complex equations as,

$$
\begin{aligned}
& \int_{t_{1}}^{t_{2}}\left[\int _ { \Omega } \left(C_{11}^{(D, k)} \varepsilon_{1}^{(0)} \frac{\partial \delta u_{r e l}}{\partial x}+C_{11}^{(F, k)} \varepsilon_{1}^{(1)} \frac{\partial^{2} \delta w_{r e l}}{\partial x^{2}}-R_{31}^{(G, k)} v(t) \frac{\partial \delta u_{r e l}}{\partial x}+R_{31}^{(H, k)} v(t) \frac{\partial^{2} \delta w_{r e l}}{\partial x^{2}}\right.\right. \\
& \quad+I^{(A, k)} \ddot{u}_{r e l} \delta u_{r e l}+I^{(A, k)} \ddot{w}_{r e l} \delta w_{r e l}-R_{31}^{(G, k)} \frac{\partial u_{r e l}}{\partial x} \delta v(t)+R_{31}^{(H, k)} \frac{\partial^{2} w_{r e l}}{\partial x^{2}} \delta v(t)-S_{33}^{(k)} v(t) \delta v(t) \\
& \left.\quad+I^{(C, k)} \frac{\partial \ddot{w}_{r e l}}{\partial x} \frac{\partial \delta w_{r e l}}{\partial x}+I^{(A, k)} \ddot{u}_{b a s e} \delta u_{r e l}+I^{(A, k)} \ddot{w}_{b a s e} \delta w_{r e l}\right) d x d y+I_{\text {tip }}^{(A)} \ddot{u}_{r e l}(L) \delta u_{r e l}(L) \\
& \quad+I_{t i p}^{(A)} \ddot{w}_{b a s e} \delta w_{r e l}(L)+I_{\text {tip }}^{(A)} \ddot{u}_{b a s e} \delta u_{r e l}(L)+I_{\text {tip }}^{(C)} \frac{\partial \ddot{w}_{r e l}}{\partial x}(L) \frac{\partial \delta w_{r e l}}{\partial x}(L)+I_{\text {tip }}^{(A)} \ddot{w}_{r e l}(L) \delta w_{r e l}(L) \\
& \left.\quad+\oint_{S}\left(n_{x} \frac{\partial M_{x x}^{(F, k)}}{\partial x} \delta w_{r e l}-n_{x} M_{x x}^{(F, k)} \frac{\partial \delta w_{r e l}}{\partial x}-n_{x} N_{x x}^{(D, k)} \delta u_{r e l}-I^{(C, k)} n_{x} \frac{\partial \ddot{w}_{r e l}}{\partial x} \delta w_{r e l}\right) d s\right] d t=0 .
\end{aligned}
$$

The superscripts $A, C, D, F, G$ and $H$ indicate properties of mass moment of inertias for first and third terms (rotary inertia), stiffness coefficients for longitudinal extension and transverse bending, piezoelectric couplings for longitudinal extension and transverse bending, respectively. Superscript $k$ indicates the layers of the bimorph. The coefficients $C_{11}, R_{31}, S_{33}, I, I_{\text {tip }}, N$ and $M$ indicate the 
stiffness coefficient, piezoelectric coupling, capacitance of the piezoelectric, mass moment of inertia of bimorph, mass moment of inertia of tip mass, internal force and moment of bimorph, respectively. Moreover, the variables $u_{r e l}, w_{\text {rel }}$ and $v(t)$ indicate the relative longitudinal and transverse displacement fields and electric voltage, respectively. The equation (1) considered the rotary inertias of the bimorph and proof mass. In this case, we ignored the rotary inertia of the bimorph component $I^{(C, k)}$ in the forthcoming mathematical derivations.

The solutions of equation (1) can be further formulated using convergent eigenfunction series forms and the solutions must meet continuity and boundary conditions of the piezoelectric bimorph beam under longitudinal extension and transverse bending effects in order to give reasonable solutions. The normalized Ritz eigenfunction series forms associated with the generalised time dependent can be prescribed as,

$$
w_{r e l}(x, t)=\sum_{r=1}^{m} \hat{\Psi}_{r}(x) w_{r}(t) \quad, \quad u_{r e l}(x, t)=\sum_{r=1}^{m} \hat{\Theta}_{r}(x) u_{r}(t) .
$$

Parameters, $\hat{\Psi}(x)$ and $\hat{\Theta}(x)$ indicate the normalised mode shapes based on the Ritz eigenfunction series which can be formulated as,

$$
\begin{gathered}
\hat{\Psi}_{r}(x)=\frac{\Psi_{r}(x)}{\left(\int_{0}^{L} I^{(A, k)} \Psi_{r}(x)^{2} d x+I_{t i p}^{(A)} \Psi_{r}(L)^{2}+I_{t i p}^{(C)}\left(\frac{d \Psi_{r}}{d x}(L)\right)^{2}\right)^{1 / 2}} \quad r=1,2, \ldots, m, \\
\hat{\Theta}_{r}(x)=\frac{\Theta_{r}(x)}{\left(\int_{0}^{L} I^{(A, k)} \Theta_{r}(x)^{2} d x+I_{t i p}^{(A)} \Theta_{r}(L)^{2}\right)^{1 / 2}} \quad r=1,2, \ldots, m .
\end{gathered}
$$

It should be noted that parameters, $\Psi_{r}(x)$ and $\Theta_{r}(x)$ are defined as the independent mode shapes of relative motions to meet the continuity conditions for the mechanical form or strain field and boundary geometry. These mode shapes can be determined using analytical solution forms [11].

Corresponding to equation (1), the orthonormality can be proved by using equation (2) in terms of equations (3) and (4) and applying the orthogonality property of the mechanical dynamic equations as,

$$
\begin{gathered}
\int_{0}^{L} I^{(A, k)} \hat{\Psi}_{r}(x) \hat{\Psi}_{q}(x) d x+I_{t i p}^{(A)} \hat{\Psi}_{r}(L) \hat{\Psi}_{q}(L)+I_{t i p}^{(C)} \frac{d \hat{\Psi}_{r}}{d x}(L) \frac{d \hat{\Psi}_{q}}{d x}(L)=\delta_{r q}, \int_{0}^{L} I^{(A, k)} \hat{\Theta}_{r}(x) \hat{\Theta}_{q}(x) d x+I_{t i p}^{(A)} \hat{\Theta}_{r}(L) \hat{\Theta}_{q}(L)=\delta_{r q}, \\
\int_{0}^{L} C_{11}^{(D, k)} \frac{d \hat{\Theta}_{r}(x)}{d x} \frac{d \hat{\Theta}_{q}(x)}{d x} d x=\omega_{r}^{(u)^{2}} \delta_{r q}, \int_{0}^{L} C_{11}^{(F, k)} \frac{d^{2} \hat{\Psi}_{r}(x)}{d x^{2}} \frac{d^{2} \hat{\Psi}_{q}(x)}{d x^{2}} d x=\omega_{r}^{(w)^{2}} \delta_{r q}
\end{gathered}
$$

where $\delta_{r q}$ is the Kronecker delta, defined as unity for $q=r$ and zero for $q \neq r$. It should be noted that the Rayleigh's damping coefficients due to longitudinal extension and transverse bending forms can also be included into equation (1). The mechanical damping coefficients can then be reduced into the mechanical damping ratios in terms of orthonormality as,

$$
C_{r q}^{(u)}=\alpha^{(u)} \delta_{r q}+\beta^{(u)} \omega_{r}^{(u)^{2}} \delta_{r q}=2 \zeta_{r}^{(u)} \omega_{r}^{(u)} \delta_{r q} \quad, \quad C_{r q}^{(w)}=\alpha^{(w)} \delta_{r q}+\beta^{(w)} \omega_{r}^{(w)^{2}} \delta_{r q}=2 \zeta_{r}^{(w)} \omega_{r}^{(w)} \delta_{r q} .
$$

Corresponding with equation (2), virtual displacement forms, $\delta u_{r}(t), \delta w_{r}(t), \delta v(t)$ can be set separately from equation (1) to obtain three independent dynamic equations. Parameters of virtual relative displacements that meet the duBois-Reymond's lemma are used to ensure that only dynamic equations have solutions. At this point, three dynamic equations of the electromechanical piezoelectric bimorph beam including the mechanical damping ratios, can be formulated after applying equations (5) and (6) and to give,

$$
\begin{aligned}
& \ddot{u}_{r}(t)+2 \zeta_{r}^{(u)} \omega_{r}^{(u)} \dot{u}_{r e l}(t)+\omega_{r}^{(u)^{2}} u_{r}(t)+P_{r}^{(u)} v(t)=-Q_{U} \ddot{u}_{\text {base }}(t) \\
& \ddot{w}_{r}(t)+2 \zeta_{r}^{(w)} \omega_{r}^{(w)} \dot{w}_{r}(t)+\omega_{r}^{(w)^{2}} w_{r}(t)+P_{r}^{(w)} v(t)=-Q_{W} \ddot{w}_{\text {base }}(t) . \\
& \hat{P}_{r}^{(u)} \dot{u}_{r}(t)+\hat{P}_{r}^{(w)} \dot{w}_{r}(t)+P_{D} \dot{v}(t)+R_{L} v(t)=0
\end{aligned}
$$


It is noted that because equation (8) has been normalised, the parameters $P_{r}^{(u)}, P_{r}^{(w)}, R_{L}$, $P_{D}, Q_{U}$ and $Q_{W}$ can be introduced as,

$$
\begin{gathered}
P_{r}^{(u)}=-\int_{0}^{L} \hat{R}_{31}^{(G, k)} \frac{d \hat{\Theta}_{r}(x)}{d x} d x, \quad P_{r}^{(w)}=\int_{0}^{L} \hat{R}_{31}^{(H, k)} \frac{d^{2} \hat{\Psi}_{r}(x)}{d x^{2}} d x, \quad \hat{P}_{r}^{(u)}=\sum_{r=0}^{m} P_{r}^{(u)}, \quad \hat{P}_{r}^{(w)}=\sum_{r=0}^{m} P_{r}^{(w)}, \\
R_{L}=\frac{1}{R_{\text {load }}}, P_{D}=-\int_{0}^{L} \hat{S}_{33}^{(k)} d x, \quad Q_{U}=\int_{0}^{L} \hat{I}^{(A, k)} \hat{\Theta}_{r}(x) d x+I_{\text {tip }}^{(A)} \hat{\Theta}_{r}(L), \quad Q_{W}=\int_{0}^{L} \hat{I}^{(A, k)} \hat{\Psi}_{r}(x) d x+I_{\text {tip }}^{(A)} \hat{\Psi}_{r}(L) .
\end{gathered}
$$

The parameter $\hat{R}_{31}$ and $\hat{S}_{33}$ can be determined in terms of the following two series and parallel connection types of the piezoelectric bimorph as,

Case I. Series connection.

a) Piezoelectric coupling for $\mathrm{X}$-poling due to transverse bending form can be formulated as,

$$
\hat{R}_{31}^{(H, k)}=-\frac{b}{2 h_{p}}\left(\frac{h_{p}{ }^{2}}{2}+\frac{h_{p} h_{s}}{2}\right) e_{31}^{(H, 1)}-\frac{b}{2 h_{p}}\left(\frac{h_{p}{ }^{2}}{2}+\frac{h_{p} h_{s}}{2}\right) e_{31}^{(H, 3)}=-\frac{b}{h_{p}}\left(\frac{h_{p}{ }^{2}}{2}+\frac{h_{p} h_{s}}{2}\right) e_{31} .
$$

b) Piezoelectric coupling for Y-poling due to longitudinal extension can also be stated as,

$$
\hat{R}_{31}^{(G, k)}=\frac{b h_{p} e_{31}^{(G, 1)}}{2 h_{p}}+\frac{b h_{p} e_{31}^{(G, 3)}}{2 h_{p}}=b e_{31}
$$

The capacitance of the piezoelectric element was considered as,

$$
\hat{S}_{33}^{(k)}=\frac{b h_{p} \varsigma_{33}^{S(1)}}{4 h_{p}^{2}}+\frac{b h_{p} \varsigma_{33}^{S(3)}}{4 h_{p}{ }^{2}}=\frac{b \varsigma_{33}^{S}}{2 h_{p}} .
$$

It should be note that the upper and lower layers of the piezoelectric bimorph will have the same material and geometrical structure where parameters $b, h_{s}$ and $h_{p}$ indicate width, substructure thickness and piezoelectric thickness of bimorph, respectively. Moreover, the piezoelectric constant $d_{31}$ can be modified into the piezoelectric constant relating charge density $e_{31}=d_{31} / s_{11}^{E}$ and the permittivity of the piezoelectric element will be $\varsigma_{33}^{S(1)}=\varsigma_{33}^{S(3)}=\varsigma_{33}^{S}$. It should be noted that $\varsigma_{33}^{S}$ is the permittivity at constant strain that can be formulated as $\varsigma_{33}^{S}=\varsigma_{33}^{T}-d_{31} e_{31}$ or $\varsigma_{33}^{S}=\varsigma_{33}^{T}-d_{31}^{2} c_{11}^{E}$ where $c_{11}^{E}=1 / s_{11}^{E}, \varsigma_{33}^{T}$ is the permittivity at constant stress and $s_{11}^{E}$ is the elastic compliance at constant electric field.

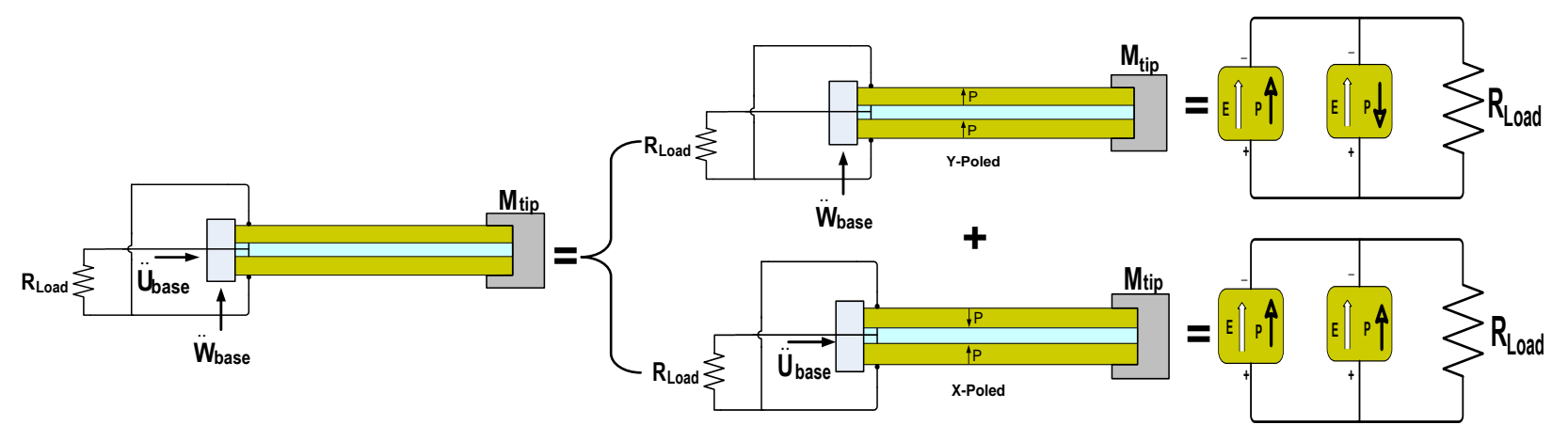

Figure 1. Cantilevered piezoelectric bimorph beam with two input base longitudinal and transverse excitations under parallel connections

Case II. Parallel connection

a) Piezoelectric coupling for X-poling due to longitudinal extension can be formulated as,

$$
\hat{R}_{31}^{(G, k)}=b e_{31}^{(G, 1)}+b e_{31}^{(G, 3)}=2 b e_{31} .
$$

b) Piezoelectric coupling for Y-poling due to transverse bending form can be formulated as, 


$$
\hat{R}_{31}^{(H, k)}=-\frac{b}{h_{p}}\left(\frac{h_{p}{ }^{2}}{2}+\frac{h_{p} h_{s}}{2}\right) e_{31}^{(H, 1)}-\frac{b}{h_{p}}\left(\frac{h_{p}{ }^{2}}{2}+\frac{h_{p} h_{s}}{2}\right) e_{31}^{(H, 3)}=-\frac{2 b}{h_{p}}\left(\frac{h_{p}{ }^{2}}{2}+\frac{h_{p} h_{s}}{2}\right) e_{31} .
$$

The capacitance of the piezoelectric element for parallel connection was given by,

$$
\hat{S}_{33}^{(k)}=\frac{b h_{p} \varsigma_{33}^{S(1)}}{h_{p}{ }^{2}}+\frac{b h_{p} \varsigma_{33}^{S(3)}}{h_{p}{ }^{2}}=\frac{2 b \varsigma_{33}^{S}}{h_{p}}
$$

It should be noted that the equation (8) also can be used for modelling the piezoelectric bimorph using either series connection or parallel connection. The connections just depend on the chosen piezoelectric couplings and also the chosen internal capacitance as given from equations (9) to (14). The sample parallel connection was shown in figure 1. Equation (8) can be solved using Laplace transformation. In this case, the multi-mode electromechanical dynamic equations of the piezoelectric bimorph with two input base motions can be formulated in terms of the frequency response functions (FRFs) of longitudinal and transverse forms and power harvesting. The first multi-mode FRF is relative longitudinal displacement with respect to input base longitudinal acceleration, which can be formulated as,

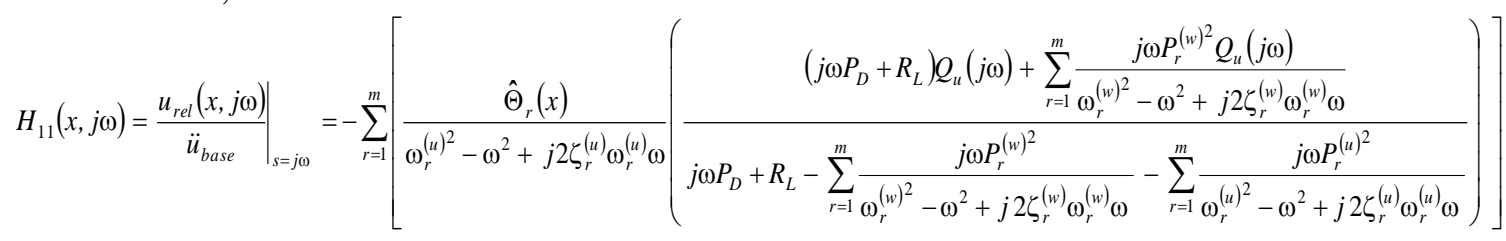

The multi-mode FRF of relative transverse displacement with respect to input base transverse acceleration can be obtained as,

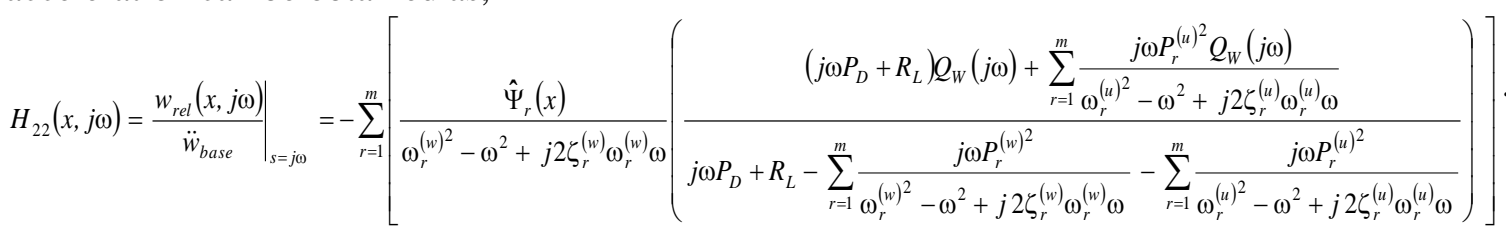

The multi-mode FRF of the relative longitudinal and transverse displacements related to the base input transverse and longitudinal accelerations can also be obtained respectively as,

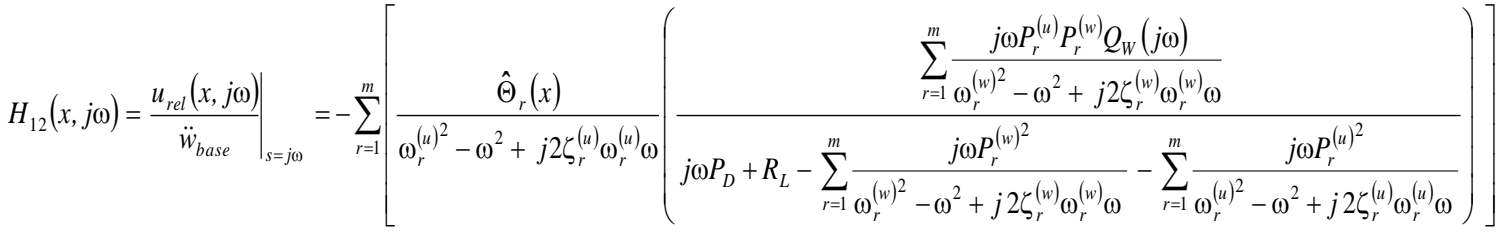

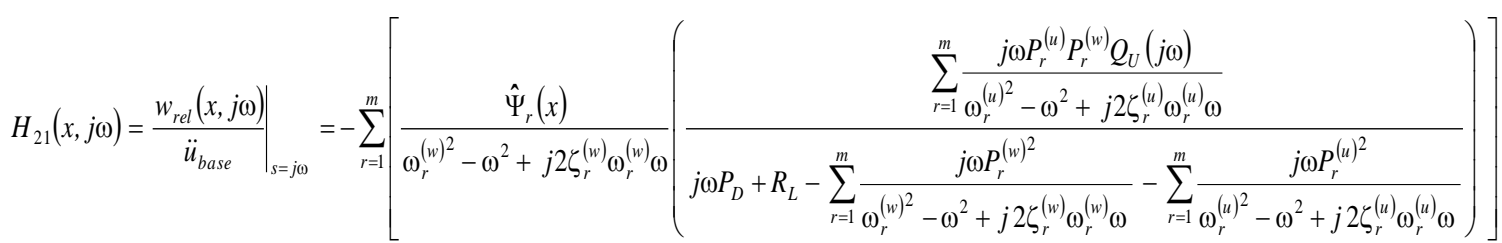

The multi-mode FRF of power harvesting relating the longitudinal acceleration can be calculated as,

$$
\left.\frac{P(j \omega)}{\left(\ddot{u}_{\text {base }}\right)^{2}}\right|_{\ddot{w}_{\text {bas }}=0}=\left[\frac{H_{31}(j \omega)}{\sqrt{R_{\text {load }}}}\right]^{2}=\left[\frac{\frac{1}{\sqrt{R_{\text {load }}}} \sum_{r=1}^{m} \frac{j \omega P_{r}^{(u)} Q_{u}(j \omega)}{\omega_{r}^{(u)^{2}}-\omega^{2}+j 2 \zeta_{r}^{(u)} \omega_{r}^{(u)} \omega}}{j \omega P_{D}+R_{L}-\sum_{r=1}^{m} \frac{j \omega P_{r}^{(w)^{2}}}{\omega_{r}^{(w)}-\omega^{2}+j 2 \zeta_{r}^{(w)} \omega_{r}^{(w)} \omega}-\sum_{r=1}^{m} \frac{j \omega P_{r}^{(u)^{2}}}{\omega_{r}^{(u)^{2}}-\omega^{2}+j 2 \zeta_{r}^{(u)} \omega_{r}^{(u)} \omega}}\right]^{2}
$$


The multi-mode FRF of power harvesting relating the transverse acceleration can be calculated as,

$$
\left.\frac{P(j \omega)}{\left(\ddot{w}_{\text {base }}\right)^{2}}\right|_{\ddot{u}_{\text {base }}=0}=\left[\frac{H_{32}(j \omega)}{\sqrt{R_{\text {load }}}}\right]^{2}=\left[\frac{\frac{1}{\sqrt{R_{\text {load }}}} \sum_{r=1}^{m} \frac{j \omega P_{r}^{(w)} Q_{W}(j \omega)}{\omega_{r}^{(w)^{2}}-\omega^{2}+j 2 \zeta_{r}^{(w)} \omega_{r}^{(w)} \omega}}{j \omega P_{D}+R_{L}-\sum_{r=1}^{m} \frac{j \omega P_{r}^{(w)^{2}}}{\omega_{r}^{(w)^{2}}-\omega^{2}+j 2 \zeta_{r}^{(w)} \omega_{r}^{(w)} \omega}-\sum_{r=1}^{m} \frac{j \omega P_{r}^{(u)^{2}}}{\omega_{r}^{(u)^{2}}-\omega^{2}+j 2 \zeta_{r}^{(u)} \omega_{r}^{(u)} \omega}}\right]^{2}
$$

The multi-mode FRF of relative longitudinal displacement can be formulated in terms of any position along the piezoelectric bimorph as,

$$
u_{r e l}(x, t)=H_{l l}(x, j \omega)\left(-\omega^{2} U_{b} e^{j \omega t}\right)+H_{l 2}(x, j \omega)\left(-\omega^{2} W_{b} e^{j \omega t}\right) .
$$

Corresponding to equation (21), the multi-mode absolute longitudinal displacement can be formulated as,

$$
u_{a b s}(x, t)=U_{b} e^{j \omega t}+H_{11}(x, j \omega)\left(-\omega^{2} U_{b} e^{j \omega t}\right)+H_{12}(x, j \omega)\left(-\omega^{2} W_{b} e^{j \omega t}\right) .
$$

The relative transverse displacement can be reformulated in terms of any position at the piezoelectric beam as,

$$
w_{r e l}(x, t)=H_{2 l}(x, j \omega)\left(-\omega^{2} U_{b} e^{j \omega t}\right)+H_{22}(x, j \omega)\left(-\omega^{2} W_{b} e^{j \omega t}\right) .
$$

The absolute transverse displacement can be reduced as,

$$
w_{a b s}(x, t)=W_{b} e^{j \omega t}+H_{21}(x, j \omega)\left(-\omega^{2} U_{b} e^{j \omega t}\right)+H_{22}(x, j \omega)\left(-\omega^{2} W_{b} e^{j \omega t}\right) .
$$

The generalised electrical potential can be formulated as,

$$
v(t)=H_{31}(j \omega)\left(-\omega^{2} U_{b} e^{j \omega t}\right)+H_{32}(j \omega)\left(-\omega^{2} W_{b} e^{j \omega t}\right) .
$$

It should be noted that $U_{b}$ and $W_{b}$ represent the input base longitudinal and transverse displacement excitations on the bimorph. Corresponding with equations (22) and (24), the equations (15) and (16) can be modified in terms of the multi-mode FRF of the absolute displacements and velocities relating the input longitudinal and transverse displacement at any position along the bimorph respectively as,

$$
\begin{aligned}
& \hat{H}_{11}^{(d i s p)}(x, j \omega)=\frac{U_{b} \mathrm{e}^{j \omega t}+u_{r e l}(x, t)}{-\omega^{2} U_{b} \mathrm{e}^{j \omega t}}=-\frac{1}{\omega^{2}}+H_{11}(x, j \omega), \quad \hat{H}_{11}^{(v e l)}(x, j \omega)=\frac{\frac{d}{d t}\left[U_{b} e^{j \omega t}+u_{r e l}(x, t)\right]}{-\omega^{2} U_{b} e^{j \omega t}}=\frac{1}{j \omega}+j \omega H_{11}(x, j \omega), \\
& \hat{H}_{22}^{(d i s p)}(x, j \omega)=\frac{W_{b} e^{j \omega t}+w_{r e l}(x, t)}{-\omega^{2} W_{b} e^{j \omega t}}=-\frac{1}{\omega^{2}}+H_{22}(x, j \omega), \quad \hat{H}_{22}^{(v e l)}(x, j \omega)=\frac{\frac{d}{d t}\left[W_{b} e^{j \omega t}+w_{r e l}(x, t)\right]}{-\omega^{2} W_{b} e^{j \omega t}}=\frac{1}{j \omega}+j \omega H_{22}(x, j \omega) .
\end{aligned}
$$

It should be noted that equations (22), (24) and (26) are applicable for analysing the absolute dynamic responses when comparing the results using the Laser Doppler Vibrometer (LDV) because the signal processing of the Vibrometer can be transferred into a digital signal through the FFT Analyzer to display the time-dependent absolute displacement, velocity, acceleration and frequency response function located at any position along the piezoelectric bimorph.

\section{Theoretical and Experimental Results}

The previous section discussed the multi-mode electromechanical piezoelectric bimorph beam under two input base longitudinal and transverse excitations. In this section, we discuss the validations between theoretical and experimental results based on the chosen properties of the piezoelectric bimorph with centre brass shim. The material properties for the piezoelectric bimorph beam were based on the PZT PSI-5A4E from Piezo Systems, INC. including brass material as shown in table 1. The piezoelectric bimorph was a cantilevered model (fixed-free model) with the generated input motion from a B \& K exciter type 4809 which was connected to the B \& K impedance head type 8001 for measuring the acceleration. Moreover, the output dynamic responses were measured by using a laser digital vibrometer Polytec PDV 100. In this case, all measurements including the electric voltage from piezoelectric component were connected to the B \& K FFT Analyzer 3560B and the results obtained can be displayed through computer screen. The complete experiment setup can be shown in 
Figure 2. Furthermore, the validation results shown here were based on the suggested formulations and experimental studies to show the transverse dynamic displacement and power harvesting around the fundamental frequency or first mode with varying resistances of the parallel connections of the piezoelectric bimorph beam.

Table 1. Characteristic properties of the piezoelectric bimorph system.

\begin{tabular}{lllllc}
\hline Material properties & Piezoelectric & Brass & Geometry properties & Piezoelectric & Brass \\
\hline Young's modulus, $\bar{Q}_{11}(\mathrm{GPa})$ & 66 & 105 & Length,$L(\mathrm{~mm})$ & 30.1 & 30.1 \\
Density, $\rho\left(\mathrm{kg} / \mathrm{m}^{3}\right)$ & 7800 & 9000 & Thickness, $h(\mathrm{~mm})$ & $0.19(\mathrm{each})$ & 0.13 \\
Piezoelectric constant, $d_{31}(\mathrm{pm} / \mathrm{V})$ & -190 & - & Width, $b(\mathrm{~mm})$ & 6.4 & 6.4 \\
Permittivity, $\varsigma_{33}^{T}(\mathrm{~F} / \mathrm{m})$ & $1800 \varsigma_{\mathrm{o}}$ & - & First coefficient $I_{\text {tip }}^{(A)}(\mathrm{kg})^{\dagger}$ & 0.0022 \\
permittivity of free space, $\varsigma_{\mathrm{o}}(\mathrm{pF} / \mathrm{m})$ & 8.854 & - & Third coefficient $I_{\text {tip }}^{(C)}\left(\mathrm{kg} \mathrm{m}^{2}\right)^{\dagger}$ & $7.3743 \times 10^{-9}$ \\
\hline
\end{tabular}

$\dagger$ Calculated according to the geometry and material properties of tip mass and the rotary inertia at centre of gravity of tip mass coincided with the end of bimorph length as shown in figures 1 and $2 b$
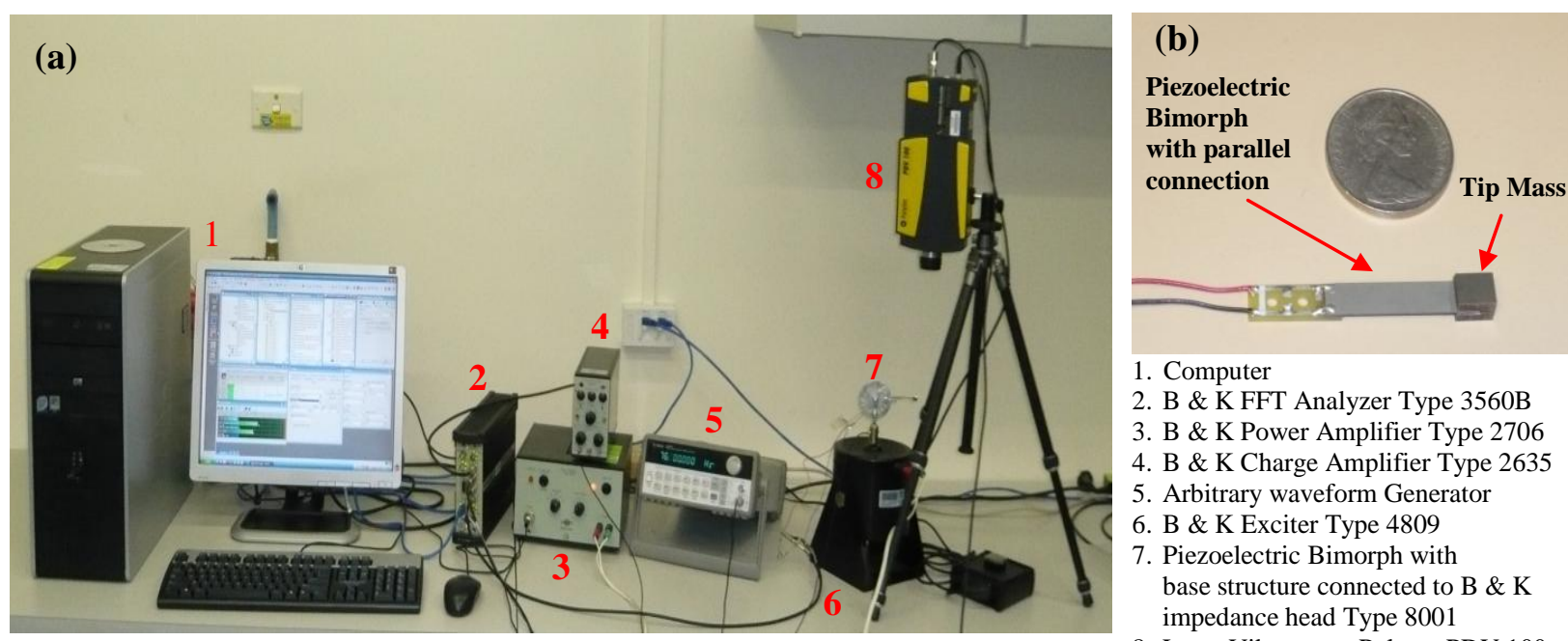

Figure 2. (a) Experimental Setup and (b) Piezoelectric bimorph beam with tip mass under parallel connection

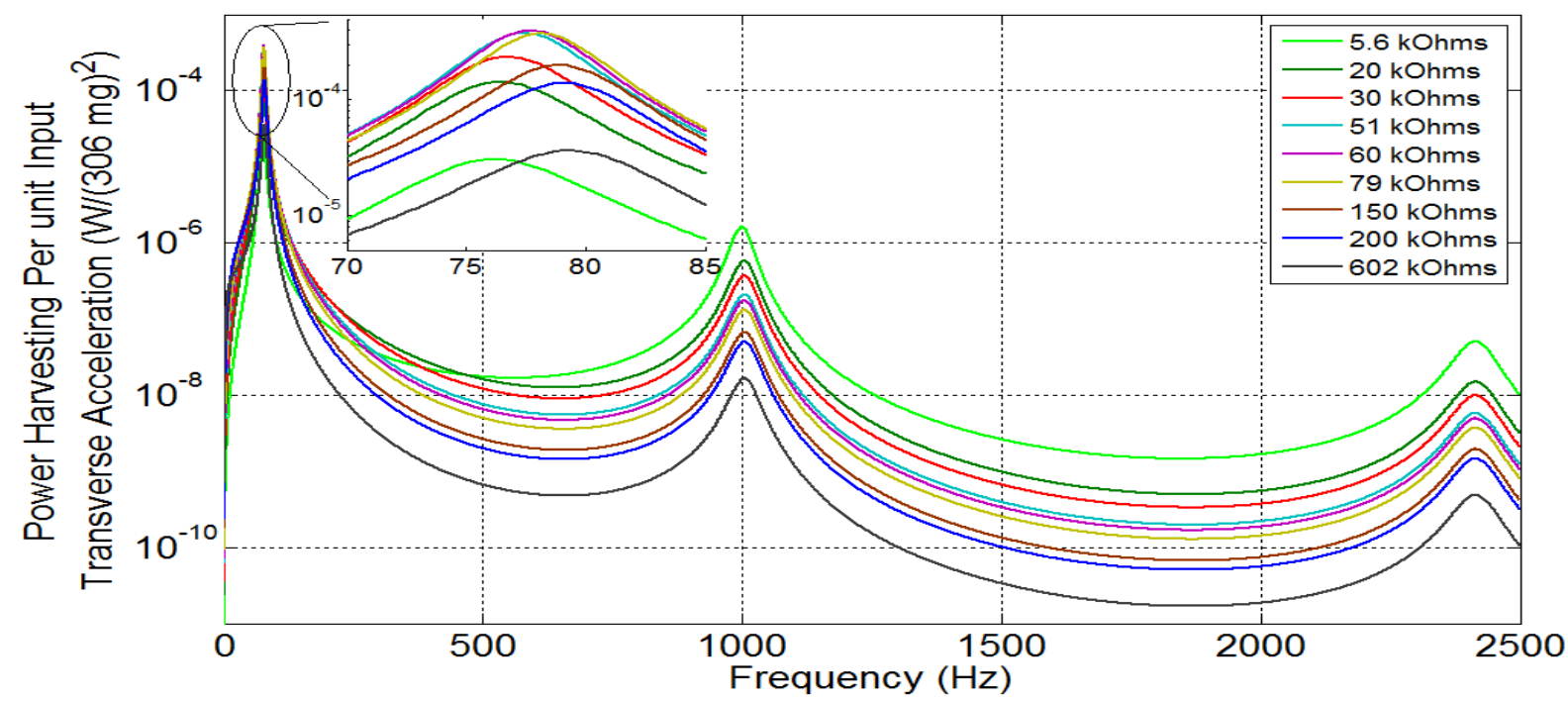

Figure 3. The first three modes of FRF power harvesting with varying load resistances 

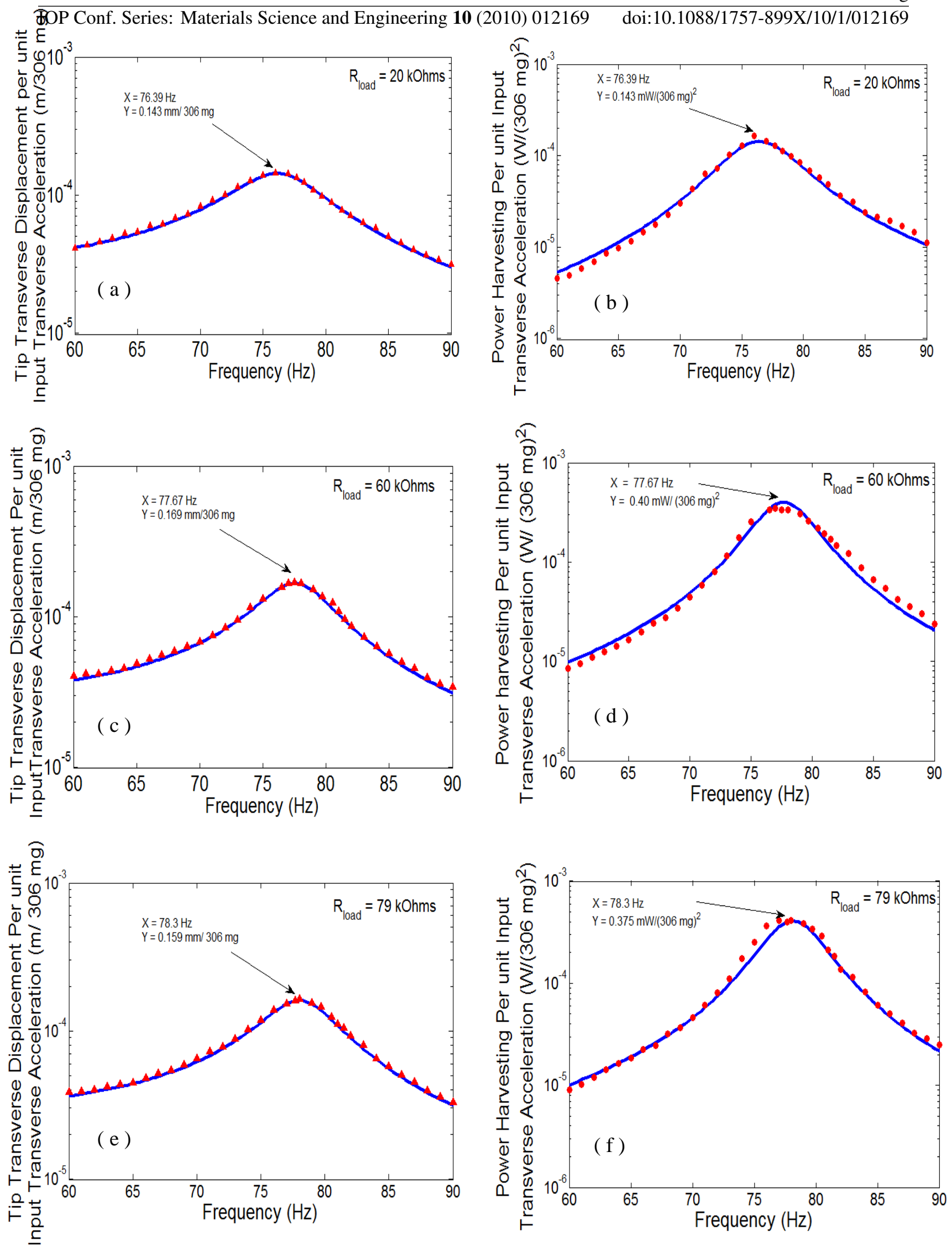

Figure 4. Comparison between theoretical and experimental results; (a) FRF tip transverse displacement and (b) FRF power harvesting for $20 \mathrm{k} \Omega$, (c) FRF tip transverse displacement and (d) FRF power harvesting for 60 $\mathrm{k} \Omega$, (e) FRF tip transverse displacement and (f) FRF power harvesting for $79 \mathrm{k} \Omega$. 
As can be seen from figure 3, the power harvesting FRF was modelled according to the first three modes due to input transverse acceleration of the bimorph base. The slight changes of power amplitudes around the first resonance mode are due to the chosen varying load resistances as shown in the enlarged viewed in figure 3. Each resonance as shown in figure 3 seems to shift frequency from $76.08 \mathrm{~Hz}$ to $79.26 \mathrm{~Hz}$ as the load resistance changes. This indicates that the load resistances can affect the power harvesting amplitude and the frequency behaviour. It is noted that when the load resistance approach zero $R_{\text {load }} \rightarrow 0$, the parallel connections of the bimorph tends to be a short circuit. In fact, the short circuit in the experimental study is only applicable to set the lowest value of resistance as possible to obtain the power harvesting magnitude in the first resonance of the frequency domain. Meanwhile, when the load resistance is approaching infinity $R_{\text {load }} \rightarrow \infty$ or the highest value, the connections of the bimorph tends to be open circuit. As far as we observed in this case, the parallel connections of the piezoelectric bimorph can be set to short circuit with the chosen load resistance around $5.6 \mathrm{k} \Omega$ and the power harvesting magnitude reached $0.0305 \mathrm{~mW} /(306 \mathrm{mg})^{2}$ whereas the open circuit of the piezoelectric bimorph was achieved by setting the load resistance around $602 \mathrm{k} \Omega$ and the resulting power harvesting obtained was around $0.0364 \mathrm{~mW} /(306 \mathrm{mg})^{2}$. In this case, the power harvesting results from the short to open circuits indicated the lowest values compared with other chosen resistances around the frequency domain. Similar trends were also reported in [12] using the design fabrication model of the aluminum nitride (AlN) based piezoelectric material and in [13] using the standard synchronized switch harvesting on inductor DC (SSHI-DC) for energy harvesting circuits. It should be noted that power harvesting results reported here represent Watts per unit square input base transverse acceleration. The input base transverse acceleration on the bimorph was $3 \mathrm{~m} / \mathrm{s}^{2}$ which is equivalent to $306 \mathrm{mg}\left(1 \mathrm{~g}=\right.$ gravitational acceleration $\left.9.81 \mathrm{~m} / \mathrm{s}^{2}\right)$. Furthermore, the first three highest power harvesting results around the first resonance with load resistances of $51 \mathrm{k} \Omega, 60 \mathrm{k} \Omega$ and $79 \mathrm{k} \Omega$ were $0.38 \mathrm{~mW} /(306 \mathrm{mg})^{2}$ at $77.35 \mathrm{~Hz}, 0.40 \mathrm{~mW} /(306 \mathrm{mg})^{2}$ at $77.67 \mathrm{~Hz}$ and $0.375 \mathrm{~mW} /(306$ $\mathrm{mg})^{2}$ at $78.3 \mathrm{~Hz}$, respectively. As can be seen, the frequencies around the first resonance tend to shift slightly with the change of the load resistances.

In this section, the comparison results between the theoretical and experimental studies are shown. The FRF transverse dynamic displacement and power harvesting according to the chosen load resistances are shown in figure 4 . It can be seen from figure $4 a$ that the transverse dynamic displacement gave very close values between the theoretical and experimental results. The comparisons obtained in this case were based on the tip absolute dynamic displacements per unit input base transverse acceleration because in the experimental results, the signal captured from the Laser Vibrometer and Analyzer indicates the time-dependent absolute dynamic displacement. Moreover, the mechanical damping ratios can be determined mathematically, but the chosen mechanical damping ratios from experiment are preferable to give the accurate results. As we observed that the mechanical damping ratios at first resonance $\{r=1\}$ due to longitudinal extension form $\zeta_{1}^{(u)}$ and transverse bending form $\zeta_{1}^{(w)}$ were found to be 0.03 and 0.06 , respectively. In this point, the mechanical damping ratio due to transverse bending form gave strong effect to the lower resonance whereas the mechanical damping ratio due to longitudinal extension form only affected to the higher resonances. It should be noted that the previous formulations were derived according to the coupled dynamic responses of the electromechanical dynamics equations. In such situation, the equation (26c) was used to compare the results obtained with respect to the experimental study. In a similar way, figure $4 \mathrm{c}$ and $4 \mathrm{e}$ show that the tip absolute displacement from experiments gave very good agreement with the theoretical results. Other chosen resistances as shown in figure 3 also showed very close comparison between the theoretical and experimental studies. Furthermore, the enlarged view from figure 3 showing the change in response with load resistance is again illustrated in figure 4 using three different load resistances $20 \mathrm{k} \Omega, 60 \mathrm{k} \Omega$ and $79 \mathrm{k} \Omega$. The power harvesting magnitudes with respect to frequency also indicate very close comparison between the theoretical and experimental results. As can be seen very clearly from figure $4 \mathrm{~b}$, the FRF power harvesting per unit square input base transverse acceleration 
gave very similar trends of both the theoretical and experimental results with power around 0.143 $\mathrm{mW} /(306 \mathrm{mg})^{2}$ with load resistance of $20 \mathrm{k} \Omega$. Once again the results of power and resonance shift as the load resistance changes. This confirms that the load resistance affect the power harvesting domain and frequency response functions.

\section{Conclusion}

This paper has presented the multi-mode electromechanical dynamic equations of a cantilevered piezoelectric bimorph beam with two input base accelerations according to the weak form of the Hamiltonian's principle. The equations derived can be used to model both series and parallel connections of the piezoelectric bimorph. In this case, we only considered the input transverse base acceleration of the bimorph with parallel connections as presented in this paper. The trend of FRF power harvesting and tip absolute dynamic displacement under varying load resistances showed very good agreement between theoretical and experimental results as investigated around the first mode. The resonances of power harvesting and absolute tip transverse displacement for the first mode shifted as the load resistance changed. As a result, the response magnitudes also changed where the input base transverse acceleration onto bimorph was kept a constant value of $3 \mathrm{~m} / \mathrm{s}^{2}$. The chosen mechanical damping ratios were found to be important when comparing between the theoretical and experimental study for predicting power harvesting magnitude and dynamic displacement. In was noted that the previous formulations were derived under the mechanical longitudinal and transverse forms. Therefore, the mechanical damping ratios at first resonance $\{r=1\}$ due to longitudinal extension form $\zeta_{1}^{(u)}$ and transverse bending form $\zeta_{1}^{(w)}$ can be preferable to be determined according to the experimental results which were found to be 0.03 and 0.06 , respectively. It was observed that the mechanical damping ratio due to transverse bending gave strong influence to the lower resonances for predicting the FRF magnitudes as similar to previous results whereas the mechanical damping ratio due to longitudinal extension form was found to only affect the higher resonances as discussed in detail by [11] using the same dimension structure but different geometry of tip mass and connection type with this paper. Finally, the FRF of the electromechanical piezoelectric bimorph system shows the benefit for simulation of the conversion of ambient vibration response into electrical power for the use of wireless sensor node for condition machine monitoring.

\section{References}

[1] Beeby S P, Torah R N , Tudor M J, Glynne-Jones P, O'Donnell T, Saha C R and Roy S 2007 J. Micromech. Microeng. 17 1257-1265

[2] Hoffmann D, Folkmer B and Manoli Y 2009 J. Micromech. Microeng. 19094001

[3] Priya S 2007 J. Electroceram. 19 167-84

[4] Anton S R and Sodano H A 2007 Smart Mater.Struct. 16 R1-21

[5] Sodano H A, Lloyd J and Inman D J. 2006 Smart Mater.Struct. 15 1211-1216

[6] Dutoit NE, Wardle B L and Kim S G 2005 J. Integr. Ferroelectr. 71 121-60

[7] Liao Y and Sodano H A 2008 Smart Mater.Struct. 17065026

[8] Goldschmidtboeing F and Woias P 2008 J. Micromech. Microeng. 18104013

[9] Erturk A and Inman D J 2009 Smart Mater.Struct. 19 1-18

[10] Lumentut M F and Howard I M 2009 Proc. ASME conf. on Smart Materials, Adaptive Structures and Intelligent Systems, Oxnard, California, USA

[11] Lumentut M F and Howard I M 2010 ASME J. Vib. Acoust. under review

[12] Elfrink R, Kamel T M, Goedbloed M, Matova S, Hohlfeld D, van Andel Y and van Schaijk R 2009 J. Micromech. Microeng. 19094005

[13] Neubauer M, Krack M and Wallaschek J 2010 J. Smart Mater. Struct. 19025001 